\title{
Other marine invertebrates collected by the diving expedition DA1/98 in the Dampier Archipelago, Western Australia
}

\author{
Mark Salotti, Jane K. Griffith* and Jane Fromont \\ Department of Aquatic Zoology (Marine Invertebrates), Western Australian Museum, \\ Francis Street, Perth, Western Australia 6000, Australia \\ email: mark.salotti@museum.wa.gov.au \\ ${ }^{*} \mathrm{c} /$ o jane.fromont@museum.wa.gov.au \\ jane fromont@museum.wa.gov.au
}

The Marine Invertebrates Section (MIZ) of the Department of Aquatic Zoology at the Western Australian Museum (WAM) has responsibility for all marine invertebrate phyla except Crustacea, Mollusca and Vermes. On the Woodside Dampier Expedition in October 1998, the groups targeted for thorough systematic collection as determined by the WAM and which are the responsibility of the MIZ Section, Department of Aquatic Zoology, were: scleractinian corals, sponges, and echinoderms. However, some additional groups for which the MIZ Section has responsibility were collected, principally belonging to the Coelenterata (Table 1). This material was collected in a random manner when time permitted and, therefore, cannot be used as comparative data between stations.

The majority of the material in Table 1 was collected by Jane Griffith, who also fixed and preserved the specimens using standard techniques required for taxonomic identification. Jane Griffith identified the soft corals except Alertigorgia, which was identified by Dr. Katherina Fabricius. Either Jane Griffith or Jane Fromont allocated the remaining coelenterate material to class or ordinal groups. The brachiopod (Z3615) was identified by Dr Robert Craig.

This list is presented to complete the documentation of material collected on the first diving expedition (DA1/98) that is held in the MIZ Section of the WAM. The other material has been documented in a number of publications: the Scleractinia (Griffith, 2004), Echinodermata (Marsh and Morrison, 2004) and Porifera (Fromont, 2003, 2004).

It is unlikely that the coelenterate material in Table 1 will be identified to species in the near future as no specialists in these groups are available at present to undertake the identifications. However this is a valuable collection that we hope to have examined at some future time.

\section{REFERENCES}

Fromont, J. (2003). Porifera (sponges) in the Dampier Archipelago: taxonomic affinities and biogeography. In Wells, F.E., Walker, D.I. and Jones, D.S. (eds), Proceedings of the Eleventh International Marine Biological Workshop: The Marine Flora and Fauna of Dampier, Western Australia: 405-417. Western Australian Museum, Perth.

Fromont, J. (2004). Porifera (sponges) of the Dampier Archipelago, Western Australia: habitats and distributions. In Jones, D.S. (ed.), Report on the Results of the Western Australia Museum/Woodside Energy Ltd. Partnership to explore the Marine Biodiversity of the Dampier Archipelago, Western Australia, 1998-2002. Records of the Western Australian Museum, Supplement 66: 69-100.

Griffith, J.K. (2004). Scleractinian corals collected during 1998 from the Dampier Archipelago, Western Australia. In Jones, D.S. (ed.), Report on the Results of the Western Australia Museum/Woodside Energy Ltd. Partnership to explore the Marine Biodiversity of the Dampier Archipelago, Western Australia, 1998-2002. Records of the Western Australian Museum, Supplement 66: 101-120.

Marsh, L.M. and Morrison, S.M. (2004). Echinoderms of the Dampier Archipelago, Western Australia. In Jones, D.S. (ed.), Report on the Results of the Western Australia Museum/Woodside Energy Ltd. Partnership to explore the Marine Biodiversity of the Dampier Archipelago, Western Australia, 1998-2002. Records of the Western Australian Museum, Supplement 66: 293-342. 
Table 1 Material collected by diving expedition DA1/98 in the Dampier Archipelago, Western Australia, not included in Griffith (2004), Fromont (2003, 2004) or Marsh and Morrison (2004) and held in the MIZ Section, Department of Aquatic Zoology, Western Australian Museum.

\begin{tabular}{|c|c|c|}
\hline Database number & Identification & Station \\
\hline \multicolumn{3}{|c|}{ Coelenterata (soft corals and sea fans) } \\
\hline Z4538 & Anthozoa: Alcyonacea: Nephtheidae: Capnella & 11 \\
\hline Z4541 & Anthozoa: Alcyonacea: Nephtheidae: Capnella & 2 \\
\hline $\mathrm{Z} 3540$ & Anthozoa: Alcyonacea: Nephtheidae: Dendronephthya spp & 15 \\
\hline $\mathrm{Z} 4501$ & Anthozoa: Alcyonacea: Nephtheidae: Dendronephthya & 15 \\
\hline $\mathrm{Z} 4502$ & Anthozoa: Alcyonacea: Nephtheidae: Dendronephthya & 11 \\
\hline Z4507 & Anthozoa: Alcyonacea: Nephtheidae: Dendronephthya & 4 \\
\hline Z4516 & Anthozoa: Alcyonacea: Nephtheidae: Dendronephthya & 15 \\
\hline $\mathrm{Z} 4521$ & Anthozoa: Alcyonacea: Nephtheidae: Dendronephthya & 15 \\
\hline Z4524 & Anthozoa: Alcyonacea: Nephtheidae: Dendronephthya & 3 \\
\hline $\mathrm{Z} 4537$ & Anthozoa: Alcyonacea: Nephtheidae: Dendronephthya & 11 \\
\hline Z4539 & Anthozoa: Alcyonacea: Nephtheidae: Dendronephthya & 11 \\
\hline $\mathrm{Z} 4560$ & Anthozoa: Alcyonacea: Nephtheidae: Dendronephthya & 13 \\
\hline $\mathrm{Z} 4568$ & Anthozoa: Alcyonacea: Nephtheidae: Dendronephthya & 4 \\
\hline $\mathrm{Z} 4513$ & Anthozoa: Alcyonacea: Nephtheidae: Stereonephthya & 13 \\
\hline $\mathrm{Z} 4571$ & Anthozoa: Alcyonacea: Nephtheidae: Stereonephthya & 4 \\
\hline $\mathrm{Z} 4525$ & Anthozoa: Alcyonacea: Alcyoniidae: cf. Alcyonium & 3 \\
\hline $\mathrm{Z} 4514$ & Anthozoa: Alcyonacea: Alcyoniidae: Cladiella & 13 \\
\hline $\mathrm{Z} 4544$ & Anthozoa: Alcyonacea: Alcyoniidae: Cladiella & 10 \\
\hline Z3504 & Anthozoa: Alcyonacea: Alcyoniidae: Lobophytum & 2 \\
\hline Z3616 & Anthozoa: Alcyonacea: Alcyoniidae: Lobophytum & 10 \\
\hline $\mathrm{Z} 3617$ & Anthozoa: Alcyonacea: Alcyoniidae: Lobophytum & 10 \\
\hline $\mathrm{Z} 3695$ & Anthozoa: Alcyonacea: Alcyoniidae: Lobophytum & 11 \\
\hline $\mathrm{Z} 3704$ & Anthozoa: Alcyonacea: Alcyoniidae: Lobophytum & 12 \\
\hline $\mathrm{Z} 3787$ & Anthozoa: Alcyonacea: Alcyoniidae: Lobophytum & 14 \\
\hline Z3901 & Anthozoa: Alcyonacea: Alcyoniidae: Lobophytum & 17 \\
\hline Z4511 & Anthozoa: Alcyonacea: Alcyoniidae: Lobophytum & 3 \\
\hline $\mathrm{Z} 4523$ & Anthozoa: Alcyonacea: Alcyoniidae: Lobophytum & 3 \\
\hline $\mathrm{Z} 4540$ & Anthozoa: Alcyonacea: Alcyoniidae: Lobophytum & 17 \\
\hline Z4555 & Anthozoa: Alcyonacea: Alcyoniidae: Lobophytum & 17 \\
\hline $\mathrm{Z} 4577$ & Anthozoa: Alcyonacea: Alcyoniidae: Lobophytum & 12 \\
\hline $\mathrm{Z} 3516$ & Anthozoa: Alcyonacea: Alcyoniidae: Sarcophyton & 3 \\
\hline Z3669 & Anthozoa: Alcyonacea: Alcyoniidae: Sarcophyton & 7 \\
\hline $\mathrm{Z} 4509$ & Anthozoa: Alcyonacea: Alcyoniidae: Sarcophyton & 18 \\
\hline $\mathrm{Z} 4927$ & Anthozoa: Alcyonacea: Anthothelidae: Alertigorgia & 11 \\
\hline Z3499 & Anthozoa: Alcyonacea & 1 \\
\hline $\mathrm{Z} 3671$ & Anthozoa: Alcyonacea & 8 \\
\hline $\mathrm{Z} 3810$ & Anthozoa: Alcyonacea & 15 \\
\hline $\mathrm{Z} 3813$ & Anthozoa: Alcyonacea & 15 \\
\hline Z3814 & Anthozoa: Alcyonacea & 15 \\
\hline $\mathrm{Z} 4518$ & Anthozoa: Alcyonacea & 15 \\
\hline Z4519 & Anthozoa: Alcyonacea & 15 \\
\hline $\mathrm{Z} 4520$ & Anthozoa: Alcyonacea & 15 \\
\hline $\mathrm{Z} 4522$ & Anthozoa: Alcyonacea & 15 \\
\hline $\mathrm{Z} 4526$ & Anthozoa: Alcyonacea & 3 \\
\hline $\mathrm{Z} 4529$ & Anthozoa: Alcyonacea & 32 \\
\hline $\mathrm{Z} 4530$ & Anthozoa: Alcyonacea & 32 \\
\hline $\mathrm{Z} 4532$ & Anthozoa: Alcyonacea & 22 \\
\hline $\mathrm{Z} 4533$ & Anthozoa: Alcyonacea & 22 \\
\hline Z4534 & Anthozoa: Alcyonacea & 22 \\
\hline $\mathrm{Z} 4543$ & Anthozoa: Alcyonacea & 15 \\
\hline Z4561 & Anthozoa: Alcyonacea & 13 \\
\hline Z4562 & Anthozoa: Alcyonacea & 13 \\
\hline $\mathrm{Z} 4563$ & Anthozoa: Alcyonacea & 13 \\
\hline $\mathrm{Z} 4564$ & Anthozoa: Alcyonacea & 13 \\
\hline $\mathrm{Z} 4565$ & Anthozoa: Alcyonacea & 13 \\
\hline Z4566 & Anthozoa: Alcyonacea & 13 \\
\hline $\mathrm{Z} 4573$ & Anthozoa: Alcyonacea & 4 \\
\hline $\mathrm{Z} 4574$ & Anthozoa: Alcyonacea & 4 \\
\hline $\mathrm{Z} 4510$ & Anthozoa: Alcyonacea: Alcyoniidae: Sarcophyton & 18 \\
\hline $\mathrm{Z} 4512$ & Anthozoa: Alcyonacea: Alcyoniidae: Sarcophyton & 17 \\
\hline
\end{tabular}


Database number

Identification

Anthozoa: Alcyonacea: Alcyoniidae: Sarcophyton

Anthozoa: Alcyonacea: Alcyoniidae: Sarcophyton Anthozoa: Alcyonacea: Alcyoniidae: Sarcophyton Anthozoa: Alcyonacea: Alcyoniidae: Sarcophyton Anthozoa: Alcyonacea: Alcyoniidae: Sarcophyton Anthozoa: Alcyonacea: Alcyoniidae: Sarcophyton Anthozoa: Alcyonacea: Alcyoniidae: Sarcophyton Anthozoa: Alcyonacea: Alcyoniidae: Sinularia Anthozoa: Alcyonacea: Alcyoniidae: Sinularia Anthozoa: Alcyonacea: Alcyoniidae: Sinularia Anthozoa: Alcyonacea: Alcyoniidae: Sinularia Anthozoa: Alcyonacea: Alcyoniidae: Sinularia Anthozoa: Alcyonacea: Alcyoniidae: Sinularia Anthozoa: Alcyonacea: Alcyoniidae: Sinularia Anthozoa: Alcyonacea: Alcyoniidae: Sinularia Anthozoa: Alcyonacea: Alcyoniidae: Sinularia Anthozoa: Alcyonacea: Alcyoniidae: Sinularia Anthozoa: Alcyonacea: Alcyoniidae: Sinularia Anthozoa: Alcyonacea: Alcyoniidae: Sinularia Anthozoa: Alcyonacea: Alcyoniidae: Sinularia Anthozoa: Alcyonacea: Alcyoniidae: Sinularia Anthozoa: Alcyonacea: Alcyoniidae: Sinularia Anthozoa: Alcyonacea: Alcyoniidae: Sinularia Anthozoa: Alcyonacea: Alcyoniidae: Sinularia Anthozoa: Alcyonacea: Alcyoniidae: Sinularia Anthozoa: Alcyonacea: Xenniidae: Xenia Anthozoa: Alcyonacea: Xenniidae: Xenia Anthozoa: Alcyonacea: Xenniidae: Xenia Anthozoa: Alcyonacea: Xenniidae: Xenia Anthozoa: Alcyonacea: Xenniidae: Xenia Anthozoa: Alcyonacea: Xenniidae: Xenia Anthozoa: Alcyonacea: Gorgoniidae: Rumphella Anthozoa: Alcyonacea

$\mathrm{Z} 4556$

Z4575
Station

15

17

14

14

17

4

12

3

3

4

10

15

4

4

4

18

15

3

6

11

14

17

13

4

10

6

6

11

23

11

11

17

4

Anthozoa: Actiniaria

Anthozoa: Actiniaria

Anthozoa: Actiniaria

9

Z2967

Z2968

Z3501

Z3505

Z3506

Z3667

Z3668

Z3670

Z3697

Z3807

Z3739

Z4550

Anthozoa: Actiniaria

Anthozoa: Actiniaria

Anthozoa: Actiniaria

Anthozoa: Actiniaria

Anthozoa: Actiniaria

Anthozoa: Actiniaria

Anthozoa: Actiniaria

Anthozoa: Actiniaria

Anthozoa: Actiniaria

Anthozoa: Actiniaria

33

1

2

2

7

7

7

11

14

12

13

Anthozoa: Zoanthidea $\quad 15$

Anthozoa: Zoanthidea 3

Anthozoa: Zoanthidea $\quad 5$

Hydrozoa: Milleporidae: Millepora sp.

Hydrozoa $\quad 22$

Other marine invertebrates 